\title{
Complex antithrombotic therapy: determinants of patient preference and impact on medication adherence
}

This article was published in the following Dove Press journal:

Patient Preference and Adherence

19 November 2015

Number of times this article has been viewed

\author{
Neena S Abraham ${ }^{1,2}$ \\ Aanand D Naik ${ }^{3,4}$ \\ Richard L Street $\mathrm{Jr}^{3-5}$ \\ Diana L Castillo ${ }^{3}$ \\ Anita Deswal ${ }^{6}$ \\ Peter A Richardson ${ }^{3,4}$ \\ Christine M Hartman ${ }^{3}$ \\ George Shelton Jr ${ }^{3,4}$ \\ Liana Fraenkel ${ }^{7,8}$ \\ 'Division of Gastroenterology, \\ Mayo Clinic, Scottsdale, AZ, USA; \\ ${ }^{2}$ Divison of Healthcare Policy and \\ Research, Department of Health \\ Services Research, Rochester, MN, \\ USA; ${ }^{3}$ Center for Innovations in \\ Quality, Effectiveness, and Safety \\ at the Michael E DeBakey VA \\ Medical Center, Houston, TX, USA; \\ ${ }^{4}$ Department of Medicine, Baylor \\ College of Medicine, Houston, TX, \\ USA; ${ }^{5}$ Department of Communication, \\ Texas A\&M University, College \\ Station, TX, USA; ${ }^{6}$ Cardiology, Michael \\ E DeBakey VAMC, Houston, TX, \\ USA; ${ }^{7}$ Veterans Affairs Connecticut \\ Healthcare System, West Haven, CT, \\ USA; ${ }^{8}$ Department of Medicine, Yale \\ University, New Haven, CT, USA
}

Correspondence: Neena S Abraham Division of Gastroenterology, Mayo Clinic, 13400 East Shea Boulevard, Scottsdale, AZ 85259, USA

$\mathrm{Tel}+\mathrm{I} 4803016990$

Fax +I 48030 I 8673

Email abraham.neena@mayo.edu
Purpose: For years, older patients have been prescribed multiple blood-thinning medications (complex antithrombotic therapy [CAT]) to decrease their risk of cardiovascular events. These therapies, however, increase risk of adverse bleeding events. We assessed patient-reported trade-offs between cardioprotective benefit, gastrointestinal bleeding risk, and burden of self-management using adaptive conjoint analysis (ACA). As ACA could be a clinically useful tool to obtain patient preferences and guide future patient-centered care, we examined the clinical application of ACA to obtain patient preferences and the impact of ACA on medication adherence.

Patients and methods: An electronic ACA survey led 201 respondents through medication risk-benefit trade-offs, revealing patients' preferences for the CAT risk/benefit profile they valued most. The post-ACA prescription regimen was categorized as concordant or discordant with elicited preferences. Adherence was measured using VA pharmacy refill data to measure persistence of use prior to and 1 year following preference-elicitation. Additionally, we analyzed qualitative interviews of 56 respondents regarding their perception of the ACA and the preference elicitation experience.

Results: Participants prioritized 5-year cardiovascular benefit over preventing adverse events. Medication side effects, medication-associated activity restrictions, and regimen complexity were less important than bleeding risk and cardioprotective benefit. One year after the ACA survey, a $15 \%$ increase in adherence was observed in patients prescribed a preference-concordant CAT strategy. An increase of only $6 \%$ was noted in patients prescribed a preference-discordant strategy. Qualitative interviews showed that the ACA exercise contributed to increase inpatient activation, patient awareness of preferences, and patient engagement with clinicians about treatment decisions.

Conclusion: By working through trade-offs, patients actively clarified their preferences, learning about CAT risks, benefits, and self-management. Patients with medication regimens concordant with their preferences had increased medication adherence at 1 year compared to those with discordant medication regimens. The ACA task improved adherence through enhanced patient engagement regarding treatment preferences.

Keywords: patient preference, patient activation, medication adherence, risk-benefit communication, cardiovascular medications, gastrointestinal bleeding

\section{Introduction}

Over 13 million Americans have coronary artery disease and 12 million Americans have peripheral vascular disease requiring therapy with antithrombotic drugs, such as anticoagulants (ie, vitamin $\mathrm{K}$ antagonists and newer oral anticoagulants) and antiplatelet drugs (ie, clopidogrel, prasugrel, ticagrelor, or aspirin [ASA]) to prevent arterial and venous thromboses. ${ }^{1}$ These cardioprotective benefits must be 
weighed against the risk of an upper gastrointestinal event (UGIE), including ulceration of the stomach and duodenum with bleeding, perforation, or obstruction. ${ }^{2}$ These risks are magnified when these drugs are prescribed in dual and triple combinations (ie, complex antithrombotic therapy [CAT]) for multiple cardiovascular conditions and when clinicians fail to consider clinical risk factors such as history of UGIE, advanced age, concomitant use of nonsteroidal anti-inflammatory drugs or corticosteroids, and Helicobacter pylori infection. ${ }^{2}$

Older patients wish to be fully informed regarding medication risks and consider alternatives that align with their values and preferences., ${ }^{3,4}$ However, clinicians rarely communicate risk-benefit trade-offs in a manner that is intuitive and actionable for their patients. ${ }^{5,6}$ This population also has higher rates of self-discontinuation of CAT regimens than younger adults. ${ }^{7}$ Concern regarding adverse effects, ${ }^{8,9}$ burden of self-management, and personal perception of potential benefit often influences older patients' preferences and willingness to initiate and adhere to CAT regimens. ${ }^{5}$ Variations in older patients' adherence are attributed to the quality of patient-physician communication, patient motivation, and their participation in treatment decisions. ${ }^{10,11}$ Yet, little is known about how older patients with multiple $\mathrm{CV}$ conditions weigh the importance of benefits or harms associated with commonly prescribed CAT strategies, and how their preferences affect medication adherence. With increasing emphasis on patient-centered care, addressing these unspoken patient preferences is critical for patient satisfaction, compliance with medication regimens, and health care outcomes.

We hypothesized that an empirically validated process of preference-elicitation would result in a more patient-centered approach to decision-making for CAT regimens. ${ }^{12}$ Our criteria for patient-centeredness included: 1) generation of patients' preferences for benefits and risks of different CAT regimens (ie, prevention of myocardial infarction [MI] and cerebrovascular accidents [CVAs]) balanced against the avoidance of UGIE or intracerebral hemorrhage [ICH], and increased treatment burdens (such as number of pills, dietary changes, and restriction of physical activity) and 2) characterization of differences in CAT preferences by age. We further hypothesized that a patient-centered approach would be associated with improved adherence to CAT as measured by pharmacy refill. Finally, we sought to evaluate patients' acceptance of using this tool prior to a potential clinical encounter.

\section{Patients and methods Compliance and funding}

This study was conducted at the Michael E DeBakey VA Medical Center (MEDVAMC) and Baylor College of Medicine in Houston, TX, USA; and at the VA Connecticut Healthcare System (VA-CHS), West Haven, CT, USA; and Yale University School of Medicine, New Haven, CT, USA, with funding from the Department of Veterans Affairs Health Services and Research Merit Award IIR-08-028 (Principal Investigator: Abraham). Institutional review and approvals were obtained from Baylor College of Medicine and Yale University School of Medicine institutional review boards as well as from Research and Development committees at Michael E. DeBakey VA Medical Center and VA Connecticut Health System.

\section{Study population and recruitment strategy}

Preferences for CAT were elicited from 201 patients recruited from internal medicine and cardiology clinics. Eligible patients were 60 years or older; cognitively intact ${ }^{13}$ with adequate health literacy ${ }^{14}$ and numeracy, ${ }^{15}$ and were prescribed antithrombotic agents (ie, ASA, anticoagulants, antiplatelets) in dual or triple combinations (ie, CAT). Recruitment was performed by a sequential stepwise strategy that included screening of clinic rosters for eligible patients followed by personal letters to contact eligible subjects by physician champions (NA, AD, and LF) along with posted notices and pamphlets advertising the study. Personal letters included an opt-out telephone number for patients to decline contact regarding study participation. Patients who did not decline contact were invited to participate and scheduled for a single 45-60-minute face-to-face interview. Subjects were compensated for their time (\$10/patient). For all participating patients, written informed consent was obtained. All interviews included a presurvey questionnaire to confirm inclusion criteria and collect data regarding patient demographics, comorbidities, concomitant medications, and preferred decision-making style. ${ }^{16}$

\section{Development of the adaptive conjoint analysis}

Adaptive conjoint analysis (ACA) is a well-validated approach to measuring preferences for competing treatment options associated with difficult trade-offs and permits segmentation of the population by similar preferences. ${ }^{17,18}$ This computer-based format engages subjects, minimizes interviewer bias, and 
facilitates data collection and management. ${ }^{19}$ Unlike other preference-elicitation programs, this software is interactive and permits evaluation of a sizeable number of trade-offs without information overload or respondent fatigue using a survey matrix that measures preferences for CAT strategies based on competing attributes (risks and benefits) of each therapy. ${ }^{17}$

The survey matrix was derived from a taxonomy of patient-derived attributes for CAT therapies from focus group samples of older, multimorbid patients. ${ }^{20,21}$ Patient-derived attributes for each CAT medication strategy (anticoagulant + thienopyridine antiplatelet [ACAP], ASA + thienopyridine antiplatelet [ASAP], ASA + anticoagulant [ASAC], and triple therapy with ASA + anticoagulant + thienopyridine antiplatelet agent [TRIP]) included: 1) cardio-protective benefits (ie, 5-year CVA risk, 5-year MI risk), 2) type and likelihood of adverse events (ie, 5-year UGIE risk, 5-year ICH risk), and 3) daily burden associated with adherence (ie, number of medications required for each strategy, physical activity limitations, presence of nuisance side effects [stomach discomfort or drug-induced dyspepsia]).

In the survey, each medication attribute was represented by specific levels to reflect a range of probabilities specific to that attribute. We conducted a systematic review of the published English-language literature and used data from our prior study of CAT-related UGIE risk ${ }^{22}$ to determine the CAT attributes included in the ACA survey. All attributes were explained in lay terminology, using a standardized script, as previously described, ${ }^{19,23,24}$ and all survey items were written in plain English at a fourth- to sixth-grade level. Natural frequencies and systematic ovals were used to facilitate the patients' understanding of probabilistic data and to decrease framing bias.

\section{Study procedures}

The research coordinator who administered the computer survey used an accompanying standardized script to describe the objectives of the survey, describe the CAT regimens, explain the type of patients who might be prescribed CAT in dual and triple combination, describe the medication attributes, and provide a standardized description of the upcoming computer task. Before patients began the survey, they were advised they would be asked their preference for medication strategies that may or may not be appropriate for their personal situation, but their opinion was important to help the investigators better understand what was most important to patients who were similar to them (ie, cardiac patients prescribed CAT).
First, subjects chose the medication attribute that is most important to them. They then rated the remaining attributes relative to the one they chose as most important. The Sawtooth software (Sawtooth Software, Inc, Sequim, WA, USA) computed an initial estimate of the subject's utilities (ie, a number representing the value that respondents associate with a particular attribute) with higher utilities representing greater value. The software then presented respondents with a series of trade-off questions with a pair of options representing a specific trade-off of two attributes (ie, a conjoint task). Attributes were presented in random order to eliminate any possible ordering effects. Participants' responses to the conjoint tasks were used to refine the ACA's estimate of each respondent's utilities. Information obtained from each conjoint task was used to update utility estimates and select the next pair of options. Utility measures become more precise as subjects discriminated among competing risks and benefits in successive pairs using a 1-9 scale, (Figure 1). By completing the questionnaire, the respondent made a series of choices, revealing intrinsic preferences for the attribute(s) he/she valued most (Figure 2). Final utilities were generated by regression analysis to derive individual respondent preferences. ${ }^{17}$ Details regarding the models used to calculate ACA utilities and preferences are available on the Sawtooth Software Web site (http://www.sawtoothsoftware.com).

\section{Outcome measures}

The relative importance of CAT attributes, given the ranges in the survey, was calculated by dividing the range of each attribute (difference between levels) by the sum of ranges of all attributes and multiplying it by $100 .{ }^{17}$ These sum to 100 and reflect the extent to which the difference between levels of each attribute affects subjects' choices (Figure 2). Attribute values are rated as 0 (least important) to 100 (most important).

\section{Analysis}

Preference data derived from ACA (version 4.0, Sawtooth Software, Inc) were imported into SAS computer files (SAS Software, version 6.12, SAS Institute, Inc, Cary, NC, USA). The mean (SD) utility for each CAT prescription strategy attribute was calculated. ACA assigns a value of zero for the least preferred attribute. The relative differences between the utilities reflect the relative values respondents associate with changes in specific characteristics. Simulations were run using the market simulator of Sawtooth Software (Sawtooth Software, Inc) to determine how variation in treatment 


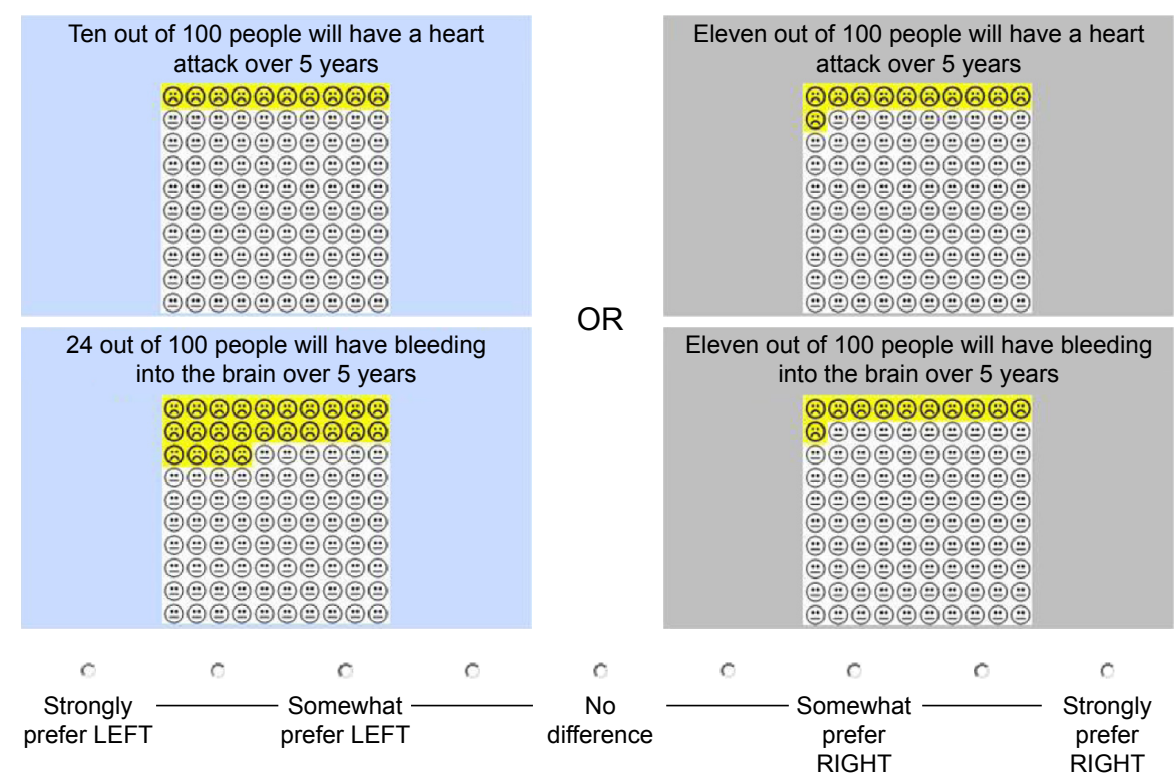

Figure I Example of adaptive conjoint analysis trade-off question.

Note: "If these two treatment options were exactly the same except for the differences above, which would you prefer - the one on the LEFT, or the one on the RIGHT?"

benefit or adverse effects influenced strength of preference for CAT-strategy attributes. ACA allows the investigator to derive preferences for a range of real and hypothetical options by specifying a level for each medication attribute. This permits assignment of varying or identical risks and/or benefits for each CAT prescription strategy. For each simulation, each respondent's file of utilities is read and a calculation is made of each respondent's relative utility for each option included in the simulation. ${ }^{23}$ The base-case scenario was constructed to model preferences for maximum $\mathrm{CV}$ survival benefit and a lowest probability of UGIE, using the first-choice model, which assumes respondents choose the option with the highest predicted utility. ${ }^{24}$ Variations in the attribute profiles included 1) a most convenient dosing schedule (minimize number of medications and reduce coagulation-level checks and physical activity restrictions); 2) a maximum CV benefit option that decreased the CVA and MI risk to lowest possible age-appropriate proportion; 3) a maximum in the age-appropriate UGIE risk, given the specific CAT strategy; and 4) a hypothetical "ideal simulation", which included a maximum cardiac benefit in terms of age-appropriate $\mathrm{CV}$ and $\mathrm{MI}$ risk reduction and minimized the UGIE risk, given the specific CAT strategy.

\section{Influence of preference-elicitation on CAT adherence}

To examine if ACA-based preference-elicitation improved patient adherence to prescribed CAT strategies, we longitu-

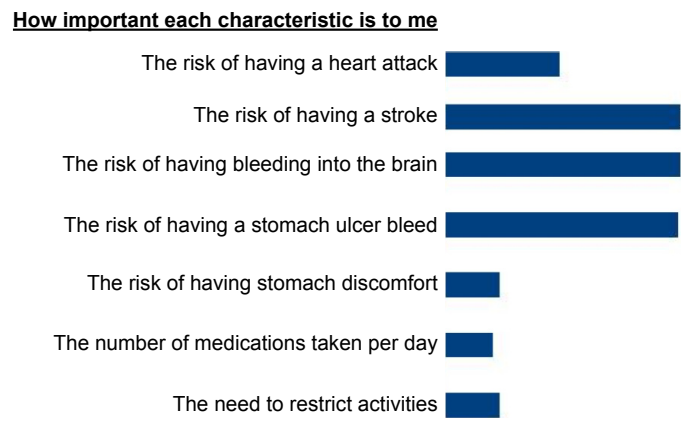

Next

Figure 2 Example of graphical representation of preferences for complex antithrombotic therapy (CAT) medication attributes.

Notes: "You have now finished all of the questions. The bars above show how important each of the seven medication characteristics are to you. The longer the bar is, the more important that characteristic was to you when you were answering the questions." 
dinally measured patients' persistence of use for 1 year following the ACA survey encounter by monitoring prescription fill data and comparing postsurvey medication adherence behavior to their adherence during the year prior to the survey encounter. We assessed adherence by determining the persistence of drug use from VA pharmacy and administrative data using methodology we have previously described. ${ }^{22,25-28}$ In this prevalent cohort of cardiac patients, the index prescription date was defined as the first prescription of an anticoagulant, antiplatelet, or ASA with a 2-year interval prior to the survey $\left(t_{0}\right)$. Total time before the survey was calculated as the time from the index date to $t_{0}$, and total time after the survey was calculated as the time from $t_{0}$ to the date of termination of the last available prescription, 365 days following $t_{0}$. The period of time before $t_{0}$ was examined to establish the patient's baseline CAT adherence pattern. The percent adherence to CAT was calculated using persistence of use, which is a validated surrogate for patient adherence $^{29}$ in pharmacy database studies. It assesses the proportion of drug supplied over the total time period being studied and measures the proportion of days for which patients have filled prescriptions. ${ }^{30}$ For each period of interest, 365 days $-t_{0}$ and $t_{0}+365$ days, we calculated the median, the 25 th and 75 th percentile values of persistency of use. We stratified the cohort by their postsurvey CAT strategy, which was categorized as concordant or discordant with that individual patient's elicited preference for a CAT strategy as demonstrated by their ACA survey. A paired $t$-test was used to compare the change in adherence pre- and postsurvey among those patients prescribed a preference-concordant or preference-discordant CAT strategies.

\section{Patient perspectives on preference- elicitation exercise (electronic ACA)}

To examine patient perception of the ACA task and possible factors associated with improved patient activation or medication adherence, we conducted qualitative interviews of 56 patients. These interviews were transcribed and analyzed for possible themes that may help further explain these patients' experiences with and possible impact from the ACA exercise.

\section{Results}

\section{Patient characteristics}

Of 388 eligible patients, 201 (51.8\%) agreed to participate, most were male (99\%), 84.1\% were Caucasian, 10.9\% were African-American, and 5\% were of other ethnicity. The mean age $(\mathrm{SD})$ was 68.6 years $(\mathrm{SD}=7$ years). Participants identified their general health status as excellent or very good $(15.9 \%)$, good $(42.8 \%)$, or fair or poor $(41.3 \%)$. The other patient characteristics are described in Table 1.

\section{Patient utilities and strength of preference for CAT attributes}

Table 2 presents patient preferences (utilities) for each prevalence estimate of CAT attributes stratified by age cohort. Figure $3 \mathrm{~A}-\mathrm{C}$ present the relative importance of particular attributes by patient cohort. All cohorts prioritized reduction of CV risk over reduction in UGIE risk. The only difference between the cohorts was those $\geq 80$ years were more strongly influenced by the risk of ICH than MI. Table 3 reports the

Table I Patient characteristics $(\mathrm{N}=202)$

\begin{tabular}{|c|c|}
\hline \multicolumn{2}{|l|}{ Demographic characteristics } \\
\hline Average age (SD) & $68.8(7.0)$ \\
\hline $60-69$ years & $62.2 \%$ \\
\hline 70-79 years & $28.9 \%$ \\
\hline$\geq 80$ years & $8.9 \%$ \\
\hline Male & $99.0 \%$ \\
\hline White & $84.1 \%$ \\
\hline Black & $10.9 \%$ \\
\hline Other race & $5.0 \%$ \\
\hline Employed & $14.9 \%$ \\
\hline Annual household income $>\$ 40,000$ & $31.8 \%$ \\
\hline College graduate & $17.9 \%$ \\
\hline Excellent or very good health status & $15.9 \%$ \\
\hline Good health status & $42.8 \%$ \\
\hline Fair or poor health status & $41.3 \%$ \\
\hline \multicolumn{2}{|l|}{ Clinical characteristics } \\
\hline History of upper gastrointestinal event & $6.5 \%$ \\
\hline History of cerebrovascular accident & $10.4 \%$ \\
\hline History of myocardial infarction & $24.9 \%$ \\
\hline Transient ischemic attack & $6.5 \%$ \\
\hline Coronary artery disease & $85.1 \%$ \\
\hline $\mathrm{PCl} / \mathrm{CABG}$ & $40.3 \%$ \\
\hline \multicolumn{2}{|l|}{ Medication characteristics } \\
\hline Current use of ACAP & $0.5 \%$ \\
\hline Current use of ASAC & $33.8 \%$ \\
\hline Current use of ASAP & $57.2 \%$ \\
\hline Current use of TRIP & $8.5 \%$ \\
\hline CAT duration $<2$ years & $24.4 \%$ \\
\hline CAT duration $3-5$ years & $29.3 \%$ \\
\hline CAT duration $>5$ years & $46.3 \%$ \\
\hline NSAID use & $9.5 \%$ \\
\hline SSRI use & $18.9 \%$ \\
\hline PPI use & $31.3 \%$ \\
\hline $\mathrm{H}_{2}$-receptor blocker use & $18.4 \%$ \\
\hline
\end{tabular}

Abbreviations: $\mathrm{SD}$, standard deviation; $\mathrm{PCl}$, percutaneous coronary intervention; CABG, coronary artery bypass graft; CAT, complex antithrombotic therapy; ACAP, combination therapy with anticoagulant and antiplatelet agent; ASAC, combination therapy with aspirin and anticoagulant; ASAP, combination therapy with aspirin and antiplatelet agent; TRIP, combination therapy with aspirin, antiplatelet agent and anticoagulant; NSAID, nonsteroidal anti-inflammatory drug; SSRI, selective serotonin reuptake inhibitor; PPI, proton pump inhibitor. 
Table 2 Patient utilities for CAT characteristics stratified by age cohort

\begin{tabular}{|c|c|c|}
\hline Characteristic & $\begin{array}{l}\text { Prevalence } \\
\text { estimate }\end{array}$ & $\begin{array}{l}\text { Utility, }{ }^{\mathrm{a}} \\
\text { mean (SD) }\end{array}$ \\
\hline \multicolumn{3}{|c|}{ Age cohort: $60-69$ years $(n=123)$} \\
\hline \multirow[t]{3}{*}{ Ml risk } & $9 \%$ & $66.1(11.0)$ \\
\hline & $10 \%$ & II.2 (6.9) \\
\hline & $13 \%$ & $-77.3(15.0)$ \\
\hline \multirow[t]{3}{*}{ CVA risk } & $7 \%$ & $71.0(8.3)$ \\
\hline & $13 \%$ & I.8 (5.3) \\
\hline & $19 \%$ & $-72.8(9.2)$ \\
\hline \multirow[t]{3}{*}{$\mathrm{ICH}$ risk } & $3 \%$ & $63.0(12.8)$ \\
\hline & $11 \%$ & $2.8(5.2)$ \\
\hline & $24 \%$ & $-65.9(13.4)$ \\
\hline \multirow[t]{3}{*}{ UGIE risk } & $2 \%$ & $40.2(6.4)$ \\
\hline & $4 \%$ & $2.6(4.0)$ \\
\hline & $9 \%$ & $-42.9(8.6)$ \\
\hline \multirow[t]{3}{*}{ Stomach discomfort } & $11 \%$ & $33.2(7.0)$ \\
\hline & $20 \%$ & $-1.6(2.3)$ \\
\hline & $22 \%$ & $-31.6(7.4)$ \\
\hline \multirow[t]{2}{*}{ Number of medications } & 2 per day & $29.4(10.4)$ \\
\hline & 3 per day & $0.9(2.1)$ \\
\hline \multirow[t]{3}{*}{ Physical activity } & No restrictions & $38.2(13.2)$ \\
\hline & in activity & \\
\hline & $\begin{array}{l}\text { Need to restrict } \\
\text { activities }\end{array}$ & $-38.2(\mid 3.2)$ \\
\hline \multicolumn{3}{|c|}{ Age cohort: $70-79$ years $(n=60)$} \\
\hline \multirow[t]{3}{*}{ Ml risk } & $10 \%$ & $62.1(9.8)$ \\
\hline & $11 \%$ & $7.5(6.7)$ \\
\hline & $13 \%$ & $-69.6(14.3)$ \\
\hline \multirow[t]{3}{*}{ CVA risk } & $7 \%$ & $79.7(10.0)$ \\
\hline & $21 \%$ & $-8.9(4.9)$ \\
\hline & $25 \%$ & $-70.7(10.7)$ \\
\hline \multirow[t]{3}{*}{$\mathrm{ICH}$ risk } & $3 \%$ & $61.7(11.9)$ \\
\hline & $11 \%$ & $2.4(5.9)$ \\
\hline & $24 \%$ & $-64.1(14.7)$ \\
\hline \multirow[t]{3}{*}{ UGIE risk } & $2 \%$ & $42.3(5.8)$ \\
\hline & $5 \%$ & I.8 (4.2) \\
\hline & $12 \%$ & $-44.1(7.4)$ \\
\hline \multirow[t]{3}{*}{ Stomach discomfort } & $11 \%$ & $36.1(9.2)$ \\
\hline & $20 \%$ & $-1.8(2.8)$ \\
\hline & $22 \%$ & $-34.3(9.0)$ \\
\hline \multirow[t]{2}{*}{ Number of medications } & 2 per day & $27.3(8.8)$ \\
\hline & 3 per day & $0.9(2.2)$ \\
\hline \multirow[t]{3}{*}{ Physical activity } & No restrictions & $39.9(13.2)$ \\
\hline & in activity & \\
\hline & $\begin{array}{l}\text { Need to restrict } \\
\text { activities }\end{array}$ & $-39.9(13.2)$ \\
\hline \multicolumn{3}{|c|}{ Age cohort: $\geq 80$ years $(n=18)$} \\
\hline \multirow[t]{3}{*}{ Ml risk } & $10 \%$ & $55.1(8.2)$ \\
\hline & $11 \%$ & $6.9(6.1)$ \\
\hline & $13 \%$ & $-62.0(10.7)$ \\
\hline \multirow[t]{3}{*}{ CVA risk } & $7 \%$ & $81.9(14.2)$ \\
\hline & $21 \%$ & $-13.2(6.9)$ \\
\hline & $25 \%$ & $-68.7(12.4)$ \\
\hline \multirow[t]{3}{*}{$\mathrm{ICH}$ risk } & $3 \%$ & $66.9(12.0)$ \\
\hline & $11 \%$ & $7.0(5.11)$ \\
\hline & $24 \%$ & $-74.0(14.0)$ \\
\hline
\end{tabular}

Table 2 (Continued)

\begin{tabular}{lll}
\hline Characteristic & $\begin{array}{l}\text { Prevalence } \\
\text { estimate }\end{array}$ & $\begin{array}{l}\text { Utility, } \\
\text { mean (SD) }\end{array}$ \\
\hline UGIE risk & $2 \%$ & $43.2(6.7)$ \\
& $7 \%$ & $1.5(5.0)$ \\
Stomach discomfort risk & $18 \%$ & $-44.8(7.1)$ \\
& $11 \%$ & $37.3(14.1)$ \\
Number of medications & $20 \%$ & $-2.8(3.8)$ \\
& $22 \%$ & $-34.5(12.5)$ \\
Physical activity & 2 per day & $25.6(9.5)$ \\
& 3 per day & $-0.03(1.6)$ \\
& No restrictions & $40.2(13.9)$ \\
& in activity & \\
& Need to restrict & $-40.2(13.9)$ \\
\hline
\end{tabular}

Note: aln this context, "utility" is a number that represents the value a respondent associates with specific CAT regimen characteristics. A higher absolute value for utility indicates a greater rating of importance for the specific characteristics.

Abbreviations: CAT, complex antithrombotic therapy; MI, myocardial infarction; CVA, cerebrovascular accident; ICH, intracerebral hemorrhage; UGIE, upper gastrointestinal event.

results of simulations conducted to examine how varying specific medication attributes influence patient preference. These simulations modeling "ideal" CV benefit-UGIE risk consistently resulted in a preference for the ASAP CAT regimen regardless of age cohort. CAT preferences did differ by age cohort when convenience and risk reduction were the dominant values (Table 3).

\section{Impact of preference-elicitation on future medication adherence}

At the time of the survey, patients were prescribed a variety of CAT regimens, including ASAP (56.8\% of patients), ASAC (33.7\% of patients), TRIP (8.9\% of patients), and ACAP ( $0.5 \%$ of patients). Each study participant was assigned a "patient-preferred CAT regimen" based on their conjoint utilities for cardioprotection, bleeding risks, and burden of self-management. When these patient-preferred CAT strategies were compared with the CAT strategy prescribed, only $20 \%$ of patients were prescribed a patientpreferred CAT strategy. Medication adherence increased by $15 \%$ in subjects prescribed CAT regimens concordant with their predicted preference, compared to a $6 \%$ increase in subjects with a prescribed regimen that was discordant with their ACA-predicted patient-preferred CAT regimen (Figure 4).

\section{Patient perspectives on preference- elicitation exercise (electronic ACA)}

Qualitative analysis revealed three major themes around patient perception of the preference-elicitation exercise 

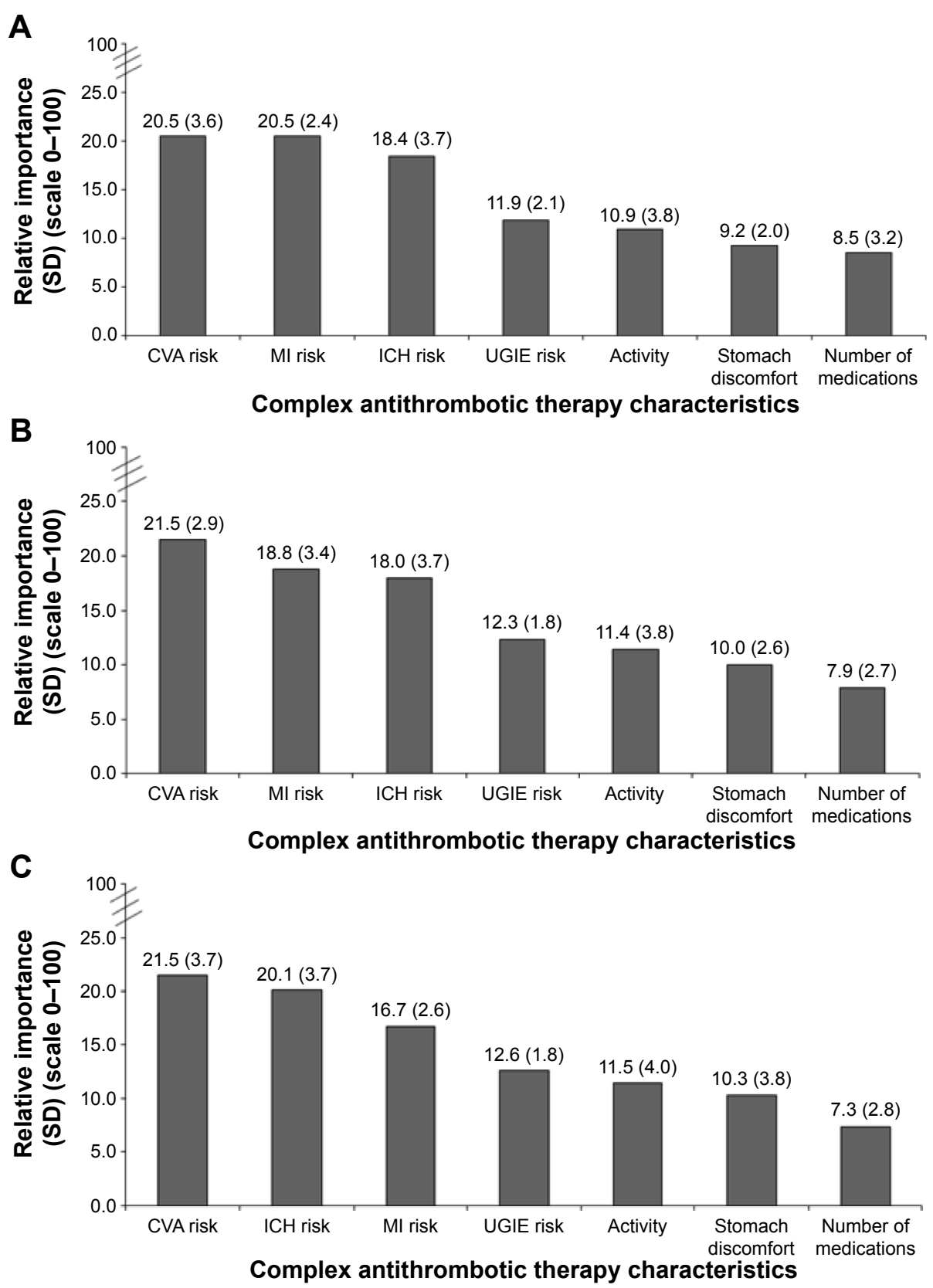

Figure 3 (A) Relative importance of CAT characteristics among patients age 60-69 years. (B) Relative importance of CAT characteristics among patients aged 70-79 years. (C) Relative importance of CAT characteristics among patients age $>80$ years.

Abbreviations: CAT, complex antithrombotic therapy; SD, standard deviation; MI, myocardial infarction; CVA, cerebrovascular accident; ICH, intracerebral hemorrhage; UGIE, upper gastrointestinal event.

(ACA task). The three major themes patients discussed were: 1) increased patient activation in their health care, 2) awareness of treatment preferences, and 3) increased engagement with clinicians (full examples in Table S1).

\section{Brief examples}

Increased patient activation in their health care:

[The patient elicitation exercise] got me thinking about stuff that I never thought about before. Maybe I'll start reading what I'm taking instead of depending on the doctor to tell me so and so [...] cuz I have stopped taking some stuff that had given me like flu symptoms. [pt 33]

Awareness of treatment preferences:

I just don't want to have a heart attack and I don't want to have a stroke, and they're both up there (on graphical representation of preferences). I don't know that much about bleeding in the brain but it just didn't sound good to me at all. I think knowing that the medicine I'm taking is going 
Table 3 Treatment preferences for CAT strategies $(\mathrm{N}=20 \mathrm{I})$ stratified by age cohort

\begin{tabular}{|c|c|c|c|c|}
\hline \multirow[t]{2}{*}{ Scenario } & \multicolumn{4}{|c|}{$\begin{array}{l}\text { Patients preferring each } \\
\text { treatment option (\%) }\end{array}$} \\
\hline & ACAP & ASAP & ASAC & TRIP \\
\hline \multicolumn{5}{|c|}{ Age cohort: $60-69$ years $(n=123)$} \\
\hline Base case & 72.2 & 4.3 & 23.5 & 0 \\
\hline Most convenient regimen ${ }^{\mathrm{a}}$ & 73.4 & 0.1 & 26.5 & 0.01 \\
\hline Most $\mathrm{CV}$ benefit ${ }^{\mathrm{b}}$ & 39.1 & 46.2 & 14.8 & 0 \\
\hline Risk of UGIE decreased ${ }^{c}$ & 1.3 & 3.8 & 95.0 & 0 \\
\hline $\begin{array}{l}\text { Most CV benefit, least } \\
\text { bleeding risk }\end{array}$ & 0.5 & 98.8 & 0.5 & 0.2 \\
\hline \multicolumn{5}{|l|}{ Age cohort: $70-79$ years $(n=60)$} \\
\hline Base case & 0.06 & 15.7 & 84.3 & 0 \\
\hline Most convenient regimen ${ }^{\mathrm{a}}$ & 0.5 & 0.8 & 98.7 & 0.01 \\
\hline Most $\mathrm{CV}$ benefit ${ }^{\mathrm{e}}$ & 16.8 & 35.7 & 47.5 & 0 \\
\hline Risk of UGIE decreased ${ }^{c}$ & 0 & 16.1 & 83.9 & 0 \\
\hline $\begin{array}{l}\text { Most CV benefit, least } \\
\text { bleeding risk }\end{array}$ & 0.3 & 99.4 & 0.3 & 0.07 \\
\hline \multicolumn{5}{|l|}{ Age cohort: $\geq 80$ years $(n=\mid 8)$} \\
\hline Base case & 0.02 & 26.2 & 73.8 & 0 \\
\hline Most convenient regimen ${ }^{\mathrm{a}}$ & 0.4 & 4.8 & 94.8 & 0.01 \\
\hline Most $\mathrm{CV}$ benefit $^{\mathrm{e}}$ & 6.8 & 56.2 & 37.0 & 0 \\
\hline Risk of UGIE decreased ${ }^{c}$ & 0 & 11.9 & 88.1 & 0 \\
\hline $\begin{array}{l}\text { Most CV benefit, least } \\
\text { bleeding risk }\end{array}$ & 0.2 & 99.6 & 0.2 & 0.05 \\
\hline
\end{tabular}

Notes: Most convenient dosing schedule $=$ two medications per day; no restrictions in activity. ${ }^{\mathrm{b}} \mathrm{MI}$ risk decreased to $9 \%$ and CVA risk decreased to $7 \%$. CUGIE risk decreased to $3 \%$. dMost CV benefit $=\mathrm{Ml}$ risk decreased to $9 \%$ and CVA risk decreased to $7 \%$; least bleeding risks $=\mathrm{ICH}$ risk decreased to $3 \%$ and UGIE risk decreased $3 \%$. ${ }^{\mathrm{eMI}}$ risk decreased to $10 \%$ and CVA risk decreased to $7 \%$. ${ }^{\mathrm{f}}$ Most $\mathrm{CV}$ benefit $=\mathrm{MI}$ risk decreased to $10 \%$ and CVA risk decreased to $7 \%$; least bleeding risks $=I C H$ risk decreased to $3 \%$ and UGIE risk decreased $3 \%$.

Abbreviations: CAT, complex antithrombotic therapy; ACAP, combination therapy with anticoagulant and antiplatelet agent; ASAC, combination therapy with aspirin and anticoagulant; ASAP, combination therapy with aspirin and antiplatelet agent; TRIP, combination therapy with aspirin, antiplatelet agent and anticoagulant; $\mathrm{CV}$, cardiovascular; ICH, intracerebral hemorrhage; MI, myocardial infarction; CVA, cerebrovascular accident; UGIE, upper gastrointestinal event.

to keep me from having either a stroke or heart attack, and so I'm going to keep taking [it]. [pt 33]

Increased patient engagement with clinicians:

I have never questioned a doctor and their medications [...] But I've, I think I've gotten to a point maybe I need to from now on. [pt 24]

\section{Discussion}

As the US population ages, the prevalence of $\mathrm{CV}$ disease among older adults has increased, affecting 30\% of men and $16 \%$ of women over age $65 .{ }^{31} \mathrm{CV}$ disease is now the leading cause of morbidity and mortality among older adults, often presenting atypically with serious health consequences. The incidence is three times higher than in younger populations. ${ }^{31}$ Death related to $\mathrm{CV}$ disease now surpasses cancer-related deaths ${ }^{31}$ and accounts for more than $60 \%$ of hospital discharges among the elderly. Secondary cardioprotection trials ${ }^{32-36}$ confirm the absolute-benefit increase of CAT to prevent MI or CVA. The Antiplatelet Trialists' Collaboration ${ }^{32}$ review of 145 studies demonstrated an absolute-benefit increase of $2.4 \%$, which corresponds to treating 42 patients with CAT to result in 1 additional secondary CVA or MI being averted.

Among the trials that included UGIE as an outcome of interest, ${ }^{32-34,36}$ a narrow therapeutic window is demonstrated by the absolute-risk increase and corresponding number needed to harm. In the CURE study, ${ }^{36}$ the absolute-benefit increase in secondary MI or CVA averted seems to outweigh the absolute increase in UGIE. However, in the MATCH study $^{34}$ fewer patients $(n=63)$ are required to be treated with CAT to result in the occurrence of an additional UGIE than are necessary to be treated to avert 1 additional CVA or MI $(\mathrm{n}=100)$, suggesting an unfavorable risk-benefit ratio. These studies highlight a narrow therapeutic window for increased absolute benefit of secondary cardioprevention compared with absolute UGIE risk. Given the strong aversion of older adults to significant adverse drug events, further research exploring patients' preferences for CAT and how these preferences impact medication adherence is greatly needed.

Conjoint analysis has proven to be an important method of evaluating patient preferences for health care and has been used to elicit preferences for treatment alternatives. ACA was deemed the most appropriate methodology for our research agenda for the following four reasons: 1) many patient-relevant attributes can be elicited and their values assessed without resulting in respondent fatigue; 2) it allows measurement of the relative importance that patients assign to specific medications based on the importance respondents assign to specific treatment-associated attributes which in turn, permits observation of how specific treatment characteristics influence an individual patient's preference for treatment choice; 3 ) it permits aggregation of individual respondents' preferences to generate an overall preference score for treatment strategies at the cohort level; and 4) it has demonstrated validity among older patients who may have mild cognitive limitations. ${ }^{24}$

We found little variation in preferences among older patients for CAT. Among all patients, CV benefit was valued more strongly than UGIE bleeding aversion. However, we did observe increasing concern regarding stroke with advancing age, best demonstrated in the stronger preferences for reduction of CVA risk and $\mathrm{ICH}$ risk among those aged 80 and older. In patients, UGIE risk associated with the CAT 


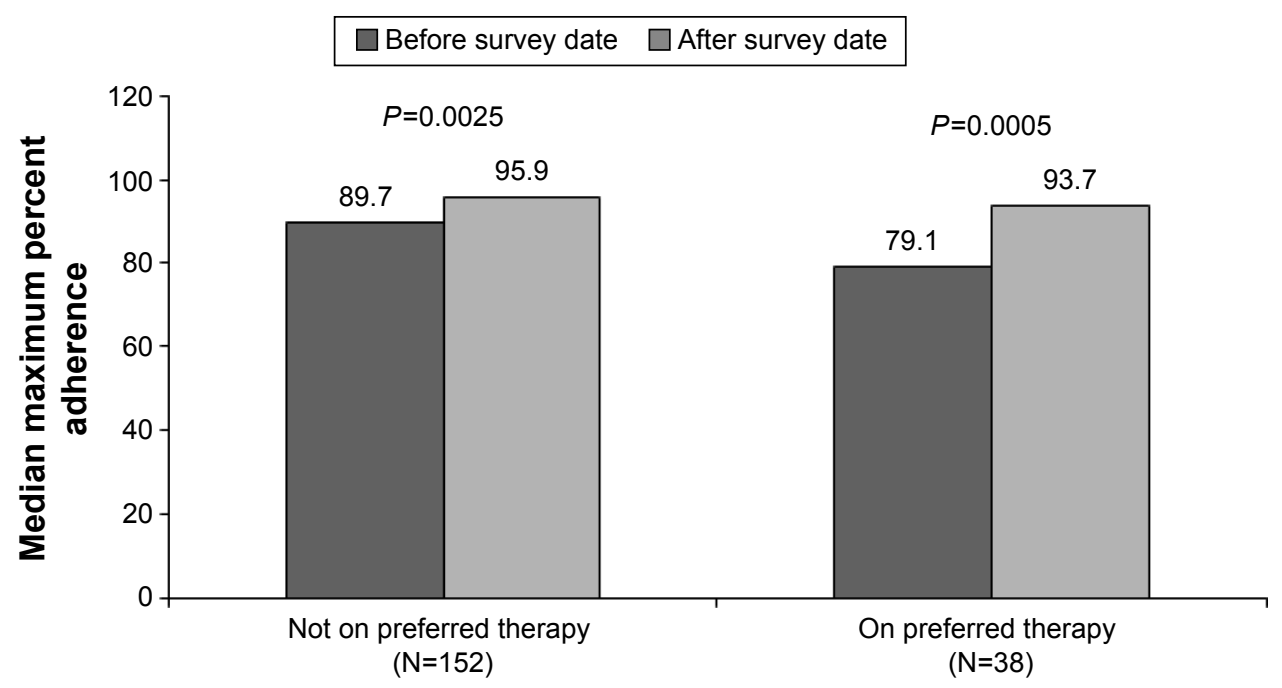

Figure 4 Change in medication adherence from baseline to I-year after completing the preference-elicitation survey, stratified by whether participants were receiving their preferred CAT therapy $(\mathrm{N}=190)$.

Abbreviation: CAT, complex antithrombotic therapy.

strategies was viewed as a "middle-of-the-pack" concern, with its relative importance being placed after potential CV benefit and the risk of $\mathrm{ICH}$, but before the importance placed on minimizing nuisance side effects and lessening the burden of medication self-management.

Our qualitative interviews revealed an important benefit of the ACA exercise was increased patient activation regarding their health care and increased patient engagement regarding treatment decisions. This increased engagement may result in improved medication adherence among patients on preferenceconcordant and possibly preference-discordant regimens as well. While only $20 \%$ were prescribed a preference-concordant CAT strategy, these patients had a 15\% increase in adherence the year following their ACA activated encounter. Even among the $80 \%$ of respondents who had been prescribed a preference-discordant CAT strategy, adherence increased by $6 \%$ following their ACA activated encounter. The benefit of a 1-time preference-elicitation activity on the 1-year adherence behavior of this cohort is surprising, especially given the response seen among those who were prescribed a preferencediscordant CAT strategy. We hypothesize that the ACA survey served as an activation moment for patients consistent with our patient-centeredness model. ${ }^{12,37}$

During this preference-elicitation exercise, conducted at the time of their provider appointment, we believe the patients were actively engaged in clarifying their preferences by explicitly learning about risks and benefits of CAT and clarifying the burden of CAT self-management. By participating in this patient-centered communication exercise, patients became 1) aware of how their cognitive and affective preferences (ie, values) related to specific clinical goals and 2) activated to discuss preferences with their physicians. This discussion confirmed and supported a behavioral strong strong behavioral intention to adhere to their prescribed regimen. Perhaps, the ACA provided explicit consideration of dominant preferences balanced against self-management burdens and potential adverse events, regardless of whether it was preference-concordant or discordant. ${ }^{12}$ Future work is required to better quantify the behavioral and perceptual mediators underpinning the improvement in medication adherence observed. ${ }^{38,39}$

Our results must be interpreted in view of the limitations of the study. Most of the patients recruited were male, as is expected with a US veteran population, thus limiting the generalizability of the findings. We did not collect detailed information regarding the patient's indication for CAT. We recognize that there are specific clinical indications (the first year post-ACS or following stent insertion) when ASAP would have superior efficacy to ASA monotherapy and in some of our participants their individual circumstances would not support the prescription of ASAP. Nonetheless, we did demonstrate, in a general $\mathrm{CV}$ population requiring $\mathrm{CAT}$, patient engagement in the decision-making process enhanced medication adherence. Since we surveyed the patients outside their patient-doctor clinical encounter, we do not know how or if the patients used the information gained from participating in the ACA exercise during their provider encounters. The failure to connect the elicited preference to the patient-doctor clinical encounter ignores the communicative context that mediates prescription discussions. 
We acknowledge the infrastructure and time requirements of using ACA as an adjunct to clinical encounters is logistically prohibitive. Future research is required to develop streamlined communication-based decision aids that can efficiently deliver the bottom-line meaning of risk-benefit and elicit preference-elicitation by encouraging patient discussion of their values, principles, and knowledge during a time-limited patient-doctor encounter. The results of the current study support the overall importance of preferenceelicitation and patient-centered communication on improvement of medication adherence.

Finally, we recognized that the way the medication attributes are described may influence the respondent's answer. To limit the potential for systematic bias in survey administration, study coordinators used standard language to ensure consistent explanations of medication-strategy attributes and the conjoint task. In addition, pilot testing at both sites allowed refinement and correction in procedures prior to recruitment.

\section{Conclusion}

Older cardiac patients valued CV benefit over the risk of GI bleeding when trading off CAT medication attributes. Despite a strong preference for dual antiplatelet therapy with ASA and a thienopyridine antiplatelet agent (ie, ASAP), only $56.8 \%$ of the cohort were prescribed this CAT strategy. Nonetheless, improved adherence was observed among patients prescribed a preference-concordant or discordant CAT strategy, suggesting the activity of preference-elicitation improved patient engagement and activation.

\section{Acknowledgments}

The authors thank Ms Barbara Corn for assistance with recruitment and data collection, and Ms Sonora Hudson for assistance with manuscript preparation.

\section{Author contributions}

Dr Abraham is the guarantor of the paper. Dr Abraham conceived, designed, and conducted the study, acquired necessary funding, interpreted the data, prepared the manuscript, and had final approval of this research. Drs Hartman and Richardson provided analytic support in programming, statistical analysis, and interpretation of results. Ms Castillo, and Drs Naik, Shelton, Street, Hartman, Richardson, Deswal, and Fraenkel assisted Dr Abraham in preparation of the manuscript. All authors contributed toward data analysis, drafting and critically revising the paper and agree to be accountable for all aspects of the work.

\section{Disclosure}

The research was supported by a grant from the Department of Veterans Affairs, Veterans Health Administration, Health Services Research and Development Service (IIR08-028); Principal Investigator: Neena S Abraham. This work was accomplished using the facilities and resources of the Michael E. DeBakey VA Medical Center Health Services Research and Development Center for Innovations in Quality, Effectiveness and Safety (CIN 13-413). The authors report no other conflicts of interest in this work.

\section{References}

1. Cannon CP, Rhee KE, Califf RM, et al; REACH Registry Investigators. Current use of aspirin and antithrombotic agents in the United States among outpatients with atherothrombotic disease (from the REduction of Atherothrombosis for Continued Health [REACH] Registry). Am J Cardiol. 2010;105:445-452.

2. Abraham NS, Hlatky MA, Antman EM, et al; ACCF/ACG/AHA. ACCF/ACG/AHA 2010 Expert Consensus Document on the concomitant use of proton pump inhibitors and thienopyridines: a focused update of the ACCF/ACG/AHA 2008 expert consensus document on reducing the gastrointestinal risks of antiplatelet therapy and NSAID use: a report of the American College of Cardiology Foundation Task Force on Expert Consensus Documents. Circulation. 2010;122:2619-2633.

3. Fraenkel L, Bogardus S, Concato J, Felson D. Preference for disclosure of information among patients with rheumatoid arthritis. Arthritis Rheum. 2001;45:136-139.

4. Naik AD, Schulman-Green D, McCorkle R, Bradley EH, Bogardus ST Jr. Will older persons and their clinicians use a shared decision-making instrument? J Gen Intern Med. 2005;20:640-643.

5. Fried TR, Tinetti ME, Towle V, O'leary JR, Iannone L. Effects of benefits and harms on older persons' willingness to take medication for primary cardiovascular prevention. Arch Intern Med. 2011;171:923-928.

6. Braddock CH III, Edwards KA, Hasenberg NM, Laidley TL, Levinson W. Informed decision making in outpatient practice: time to get back to basics. JAMA. 1999;282:2313-2320.

7. Ali RC, Melloni C, Ou FS, et al. Age and persistent use of cardiovascular medication after acute coronary syndrome: results from medication applied and sustained over time. J Am Geriatr Soc. 2009;57: 1990-1996.

8. Belcher VN, Fried TR, Agostini JV, Tinetti ME. Views of older adults on patient participation in medication-related decision making. J Gen Intern Med. 2006;21:298-303.

9. Fried TR, McGraw S, Agostini JV, Tinetti ME. Views of older persons with multiple morbidities on competing outcomes and clinical decisionmaking. J Am Geriatr Soc. 2008;56:1839-1844.

10. Baroletti S, Dell'Orfano H. Medication adherence in cardiovascular disease. Circulation. 2010;121:1455-1458.

11. Montori VM, Breslin M, Maleska M, Weymiller AJ. Creating a conversation: insights from the development of a decision aid. PLoS Med. 2007; 4:e233.

12. Naik AD. On the road to patient centeredness. Comment on: "communication and medication refill adherence". JAMA Intern Med. 2013; 173:218-219.

13. Pfeiffer E. A short portable mental status questionnaire for the assessment of organic brain deficit in elderly patients. J Am Geriatr Soc. 1975; 23:433-441.

14. Chew LD, Bradley KA, Boyko EJ. Brief questions to identify patients with inadequate health literacy. Fam Med. 2004;36:588-594.

15. Lipkus IM, Samsa G, Rimer BK. General performance on a numeracy scale among highly educated samples. Med Decis Making. 2001;21: $37-44$. 
16. Degner LF, Kristjanson LJ, Bowman D, et al. Information needs and decisional preferences in women with breast cancer. JAMA. 1997;277: 1485-1492.

17. Johnson RM. Adaptive conjoint analysis. In: Sawtooth Software, editor. Proceedings of the Sawtooth Software Conference on Perceptual Mapping, Conjoint Analysis, and Computer Interviewing. Ketchum, ID: Sawtooth Software; 1987;253-265.

18. Ryan M, Farrar S. Using conjoint analysis to elicit preferences for health care. BMJ. 2000;320:1530-1533.

19. Fraenkel L, Bogardus S, Wittnik DR. Understanding patient preferences for the treatment of lupus nephritis with adaptive conjoint analysis. Med Care. 2001;39:1203-1216.

20. Abraham NS, Castillo DL, Naik AD. NSAID-cardioprotective drug polypharmacy: patient beliefs and expectations of treatment goals. J Am Geriatr Soc. 2008;56:S49.

21. Andreas DC, Abraham NS, Naik AD, Street RL Jr, Sharf BF. Understanding risk communication through patient narratives about complex antithrombotic therapies. Qual Health Res. 2010;20:1155-1165.

22. Abraham NS, Hartman C, Richardson P, Castillo D, Street RL Jr, Naik AD. Risk of lower and upper gastrointestinal bleeding, transfusions, and hospitalizations with complex antithrombotic therapy in elderly patients. Circulation. 2013;128:1869-1877.

23. Fraenkel L, Bogardus ST, Concato J, Felson DT, Wittink DR. Patient preferences for treatment of rheumatoid arthritis. Ann Rheum Dis. 2004; 63:1372-1378

24. Fraenkel L, Bogardus ST Jr, Concato J, Wittink DR. Treatment options in knee osteoarthritis: the patient's perspective. Arch Intern Med. 2004; 164:1299-1304.

25. Abraham NS, El-Serag HB, Johnson ML, et al. National adherence to evidence-based guidelines for the prescription of nonsteroidal antiinflammatory drugs. Gastroenterology. 2005;129:1171-1178.

26. Abraham NS, El-Serag HB, Hartman C, Richardson P, Deswal A Cyclooxygenase-2 selectivity of non-steroidal anti-inflammatory drugs and the risk of myocardial infarction and cerebrovascular accident Aliment Pharmacol Ther. 2007;25:913-924.

27. Abraham NS, Hartman C, Castillo D, Richardson P, Smalley W. Effectiveness of national provider prescription of PPI gastroprotection among elderly NSAID users. Am J Gastroenterol. 2008;103:323-332.

28. Abraham NS, Hartman C, Hasche J. Reduced hospitalization cost for upper gastrointestinal events that occur among elderly veterans who are gastroprotected. Clin Gastroenterol Hepatol. 2010;8:350-356.
29. Steiner JF, Prochazka AV. The assessment of refill compliance using pharmacy records: methods, validity, and applications. J Clin Epidemiol. 1997;50:105-116.

30. Steiner JF, Koepsell TD, Fihn SD, Inui TS. A general method of compliance assessment using centralized pharmacy records. Description and validation. Med Care. 1988;26:814-823.

31. Go AS, Mozaffarian D, Roger VL, et al; on behalf of the American Heart Association Statistics Committee and Stroke. Heart disease and stroke statistics - 2013 update: a report from the American Heart Association. Circulation. 2013;127:e6-e245.

32. Collaborative overview of randomised trials of antiplatelet therapy $-\mathrm{I}$ : prevention of death, myocardial infarction, and stroke by prolonged antiplatelet therapy in various categories of patients. Antiplatelet Trialists' Collaboration. BMJ. 1994;308:81-106.

33. Anand SS, Yusuf S, Pogue J, Weitz JI, Flather M. Long-term oral anticoagulant therapy in patients with unstable angina or suspected non-Q-wave myocardial infarction: organization to assess strategies for ischemic syndromes (OASIS) pilot study results. Circulation. 1998;98: 1064-1070.

34. Diener HC, Bogousslavsky J, Brass LM, et al. Aspirin and clopidogrel compared with clopidogrel alone after recent ischaemic stroke or transient ischaemic attack in high-risk patients (MATCH): randomised, double-blind, placebo-controlled trial. Lancet. 2004;364:331-337.

35. van Es RF, Jonker JJ, Verheugt FW, Deckers JW, Grobbee DE. Aspirin and coumadin after acute coronary syndromes (the ASPECT-2 study): a randomised controlled trial. Lancet. 2002;360:109-113.

36. Yusuf S, Zhao F, Mehta SR, Chrolavicius S, Tognoni G, Fox KK. Effects of clopidogrel in addition to aspirin in patients with acute coronary syndromes without ST-segment elevation. $N$ Engl J Med. 2001;345: 494-502.

37. Abraham NS, Naik AD, Street RL Jr. Shared decision making in GI clinic to improve patient adherence. Clin Gastroenterol Hepatol. 2012;10:825-827.

38. Street RL, Elwyn G, Epstein RM. Patient preferences and healthcare outcomes: an ecological perspective. Expert Rev Pharmacoecon Outcomes Res. 2012;12:167-180.

39. Reyna VF. A theory of medical decision making and health: fuzzy trace theory. Med Decis Making. 2008;28:850-865. 


\section{Supplementary material}

Table SI Patient perception of preference-elicitation exercise $(\mathrm{N}=56)$

Domain: increased patient engagement with clinicians

"[The patient elicitation exercise] got me thinking about stuff that I never thought about before. Maybe l'll start reading what l'm taking instead of depending on the doctor to tell me so and so [...] cuz I have stopped taking some stuff that had given me like flu symptoms". [pt 33]

"I believe [the exercise] is interesting number one. It's informative. I never thought about all of this at one time. In other words, one of two things crossed my mind but never all of them. Based actually on the same thing, just a different way of putting each thing ... Makes you think”. [pt I7] "[...] the most difficult part of [the exercise] was the choice of one bad thing increasing with a decrease in a good thing happening. You know, not a good thing, but a different bad thing. Yeah, that [the trade-off] was a little difficult". [pt 47]

"I think it's good that you make me address these things ... I think there's a lot more that we need to start paying attention to". [pt 24]

Domain: increased patient awareness of treatment preferences

"[The preference elicitation exercise] gave me more insight into what I am feeling about the medications that l'm taking, and how I'm feeling about the differences between the strokes, the heart attacks, the bleeds, and things like that. To be honest about it, I hadn't really ever thought about it that much. So, this has gave me some kind of insight". [pt 25]

"I can see where the benefit of knowing these things is better than the risk of not knowing about these health conditions, because I have them and I need to be more conscious about doing the things that I need to do and that I will be on these medicines for the rest of my life ....". [pt 4]

"[The preference elicitation exercise] shows what is more important to me... As far as my activities and the results that can happen ... it shows that someone's trying to see what the patient's main concerns are". [pt 9]

"I think [the preference elicitation exercise] is something that is beneficial. It really determines how you feel about your condition and the medications you take". [pt 45]

“I just don't want to have a heart attack and I don't want to have a stroke, and they're both up there (on graphical representation of preferences). I don't know that much about bleeding in the brain but it just didn't sound good to me at all. I think knowing that the medicine l'm taking is going to keep me from having either a stroke or heart attack, and so l'm going to keep taking [it]". [pt 33]

Domain: increased activation

"My biggest concern is bleeding into the brain ... and second up is the stroke and the heart attack ... Yeah, [I] definitely think [the preference elicitation exercise] is worthwhile - to be given this information and be able to use it in my decision". [pt 36]

"I would definitely have to ask [the physician] to monitor the ones I deem necessary in [the preference elicitation exercise] ... I would question him about it and make sure he monitors it”. [pt 23]

"I am telling [the physician] my concerns when I have a checkup as far as the medications that I take". [pt 9]

"If these percentages (on graphical representation of preferences) are anywhere near right, l'm gonna be talking to my doctor a little bit better". [pt I7] "I have never questioned a doctor and their medications ... But l've, I think l've gotten to a point maybe I need to from now on". [pt 24]

"Well, if there's a decision to be made about what to do about my particular condition, this information and the way I feel on the survey would come into bearing on [the] decision that I would make with my physicians". [pt 47]

Abbreviation: pt, patient.

\section{Publish your work in this journal}

Patient Preference and Adherence is an international, peer-reviewed, open access journal that focuses on the growing importance of patient preference and adherence throughout the therapeutic continuum. Patient satisfaction, acceptability, quality of life, compliance, persistence and their role in developing new therapeutic modalities and compounds to optimize clinical outcomes for existing disease states are major areas of interest for the journal. This journal has been accepted for indexing on PubMed Central. The manuscript management system is completely online and includes a very quick and fair peer-review system, which is all easy to use. Visit http://www. dovepress.com/testimonials.php to read real quotes from published authors. 